\title{
A dahlia like pattern: A new dermoscopic sign of filiform wart
}

\author{
Afaf Khouna', Hazim Aburabie', Hanane Daflaoui ${ }^{1,2}$, Nada Zizi, Siham Dikhaye ${ }^{1,2}$ \\ ${ }^{1}$ Department of Dermatology, Mohammed VI University Hospital of Oujda, Medical School of Oujda, Mohammed First \\ University of Oujda, Morocco, ${ }^{2}$ Epidemiology, Clinical Research and Public Health Laboratory, Medical School of Oujda, \\ Mohammed First University of Oujda, Morocco
}

Corresponding author: Afaf Khouna, MD, E-mail: afaf.khouna@gmail.com

Sir,

Warts are common benign epidermal proliferations caused by various strains of human papillomavirus. Dermoscopic examination of these lesions may help to distinguish them [1]. Filiform warts are small, finger-like, and pedunculated [2]. They often appear in periorificial areas, mostly in children or in the beard area of men [3]. They are usually treated with surgical removal or cryotherapy. We report a case of a descriptive dermoscopic image of a filiform wart in a young moroccan male [2].

Our patient is a 36 years old male, without antecedents, who presented a rounded papular keratosic red lesion of the right arm (fig 1), evolving since 1 year. Dermoscopic examination found a flower-like pattern (fig 2), more precisely a dahlia-like pattern (fig 3), with a tapered fingerlike pattern (black arrow) and a keratotic white tip (red arrow) and pigmented zones (white arrow).

We have done a complete exeresis of the lesion. Histological examination showed an acanthotic epidermis surmounted by a focal parakeratotic and orthokeratotic hyperkeratosis.

Recently, a study including a large number of patients identified four dermoscopic patterns that may also coexist in a single wart: unspecific, fingerlike, mosaic and knoblike patterns. Glomerular, hairpin/dotted, and glomerular/dotted vessel morphologies were also described [1].

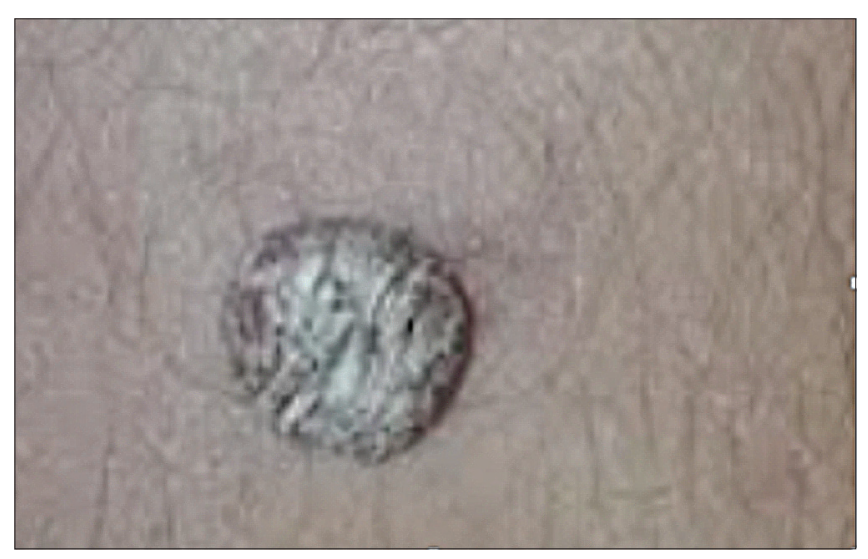

Figure 1: Papular keratosic red lesion of the right arm.

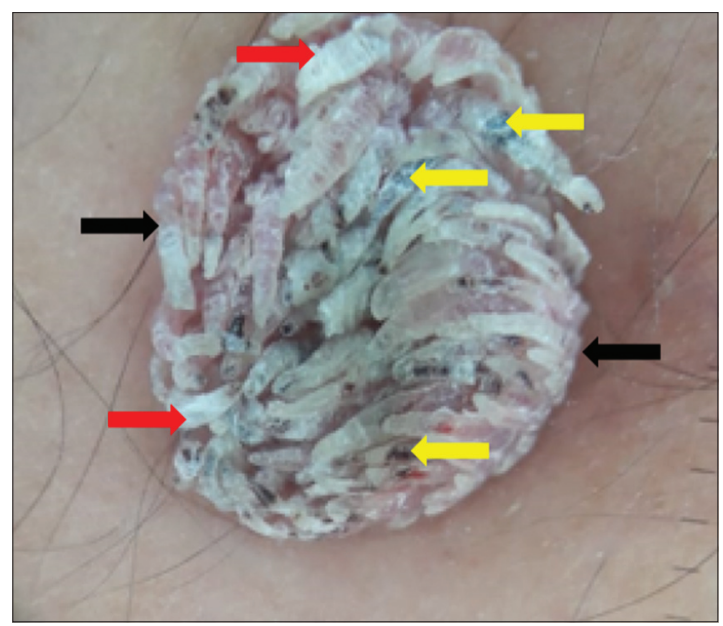

Figure 2: Dermoscopic examination found a flower-like pattern: Dahlia-like pattern. Black arrow: tapered fingerlike pattern; Red arrow: keratosic white tip; White arrow: pigmented zones.

For filiform wart, dermoscopy shows the same features as common warts, with more prevalent papillae.

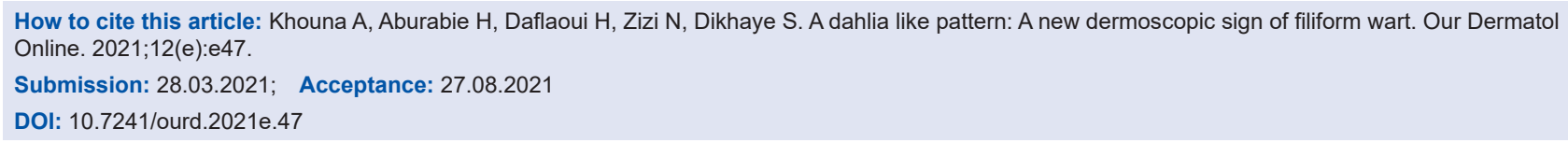


www.odermatol.com

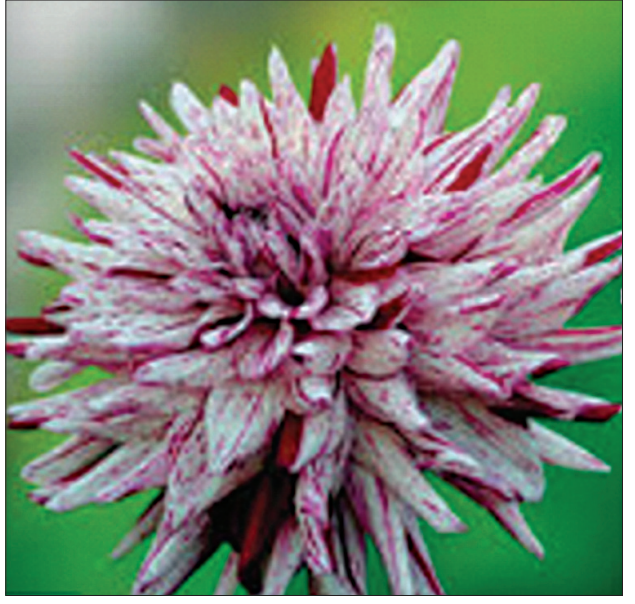

Figure 3: Dahlia flower.

Dotted vessels are detectable at the extremities of each papilla [3].

Filiform warts are often treated with surgical intervention via a "snip excision" using surgical scissors or shave removal. Additionally, snip excisions have the potential to scar and are undesirable, especially if the patient has a known history of keloid formation. Another commonly used treatment modality for filiform warts is cryotherapy. Similarly, podophyllum, cantharidin, and chemical peels may be used, but such therapies carry a significant risk of hyperpigmentation and blistering.

A combination of $5-\mathrm{FU} 5 \%$ and salicylic acid $20 \%$ achieved a greatest treatment response [2].

\section{REFERENCES}

1. Moustaide K. The dermoscope: Our hero. J Dermatol Surg Res Ther. 2018:7-8

2. Cartron AM, Blaszczak A, Trinidad JC. Combination therapy with 5 -fluorouracil and salicylic acid in a treatment-resistant case of filiform facial warts. Dermatol Ther. 2020;33:e13235.

3. Piccolo V. Update on dermoscopy and infectious skin diseases. Dermatol Pract Concept. 2019;10:e2020003.

Copyright by Afaf Khouna, et al. This is an open access article distributed under the terms of the Creative Commons Attribution License, which permits unrestricted use, distribution, and reproduction in any medium, provided the original author and source are credited.

Source of Support: Nil, Conflict of Interest: None declared. 